Int. J. Electrochem. Sci., 12 (2017) $8327-8336$

\title{
Ultrasensitive Electrochemiluminescence Sensor for Specific Detection of Rutin Based on CdS Modified Pencil Graphite Electrode
}

Ruijuan Zheng ${ }^{1,4}$, Chenhao Zhao ${ }^{1,2}$, Jianhai Zhong ${ }^{3}$,Zehai Qiu ${ }^{1,2}$, Guixiang Xie $^{1}$, Jijian Wei ${ }^{1}$, Zhibiao $\mathrm{Hu}^{1, *}$, Jiangshui Luo ${ }^{1,4, *}$

${ }^{1}$ College of Chemistry and Materials Science, Longyan University, Longyan, Fujian, 364012, China. ${ }^{2}$ Fujian Provincial Key Laboratory of Clean Energy Materials, LongYan University, Fujian LongYan

${ }^{3}$ Longyan Entry-Exit Inspection and Quarantine Bureau, Longyan, China

${ }^{4}$ Lab of Proton conductors, Longyan University, Longyan, Fujian, 364012, China

*E-mail: flyzhibiaohu@163.com, proticils@gmail.com

doi: $10.20964 / 2017.09 .20$

Received: 9 May 2017 / Accepted: 23 June 2017 / Published: 12 August 2017

In this paper, A CdS modified pencil graphite electrode(CdS/PGE) was fabricated using solvothermal route through simple procedure. The CdS/PGE showed unexpectedly electrochemiluminescent behaviour with potassium persulfate $\left(\mathrm{K}_{2} \mathrm{~S}_{2} \mathrm{O}_{8}\right)$ as a coreactant. Based on this principle that ECL was quenched when rutin was present in this system, an ECL sensor for detecting rutin had been developed. The effects of buffer solution and $\mathrm{pH}$ were studied in detail. Under the optimum conditions, a possible mechanism of ECL quenching was also clearly discussed. The linear correlation between the quenched ECL intensity and the logarithm of rutin concentration was observed over the range of $4 \times 10^{-}$ ${ }^{9} \sim 8 \times 10^{-7} \mathrm{~mol} / \mathrm{L}$ with the limit of detection (LOD) of $2 \times 10^{-9} \mathrm{~mol} / \mathrm{L}$. This proposed method was applied in the analysis of rutin in compound rutin tablets, with recoveries of $98 \% \sim 105 \%$. The experimental results demonstrated that the ECL sensor showed good stability and reproducibility.

Keywords: Electrochemiluminescence, CdS nanorods, pencil graphite electrode, rutin,

\section{$\underline{\text { FULL TEXT }}$}

(C) 2017 The Authors. Published by ESG (www.electrochemsci.org). This article is an open access article distributed under the terms and conditions of the Creative Commons Attribution license (http://creativecommons.org/licenses/by/4.0/). 